\title{
Cryptococcal Pleuritis Containing a High Level of Adenosine Deaminase in a Patient with AIDS: A Case Report
}

\author{
Yusuke Yoshino $^{a}$ Takatoshi Kitazawa $^{\mathrm{a}}$ Keita Tatsuno ${ }^{\mathrm{a}}$ Yasuo Ota ${ }^{\mathrm{b}}$ \\ Kazuhiko Koike ${ }^{a}$ \\ aDepartment of Infectious Diseases, Graduate School of Medicine, University of Tokyo, and \\ ${ }^{b}$ Department of Medicine, Teikyo University School of Medicine, Tokyo, Japan
}

\section{Established Facts}

- In cryptococcal infection, pleural effusion alone is an unusual presentation.

- High levels of adenosine deaminase in the pleural fluid are used for clinical diagnosis of tuberculous pleuritis.

\section{Novel Insights}

- High levels of adenosine deaminase in the pleural fluid can be observed in cryptococcal pleuritis.

- Longer incubation of pleural fluid should be performed in cases of pleuritis with a high adenosine deaminase level as the only significant finding.

\section{Key Words}

Cryptococcus neoformans - Pleuritis - Adenosine

deaminase $\cdot$ Pleural effusion

\begin{abstract}
Cryptococcal infection is the 4th most common opportunistic infection in patients with acquired immune deficiency syndrome (AIDS). Although pleural effusion alone is an unusual presentation, we present a case of cryptococcal pleu-
\end{abstract}

ritis in an AIDS patient which was initially difficult to discriminate from tuberculous pleuritis because of the high level of pleural adenosine deaminase (ADA). Cryptococcus neoformans was detected in the culture of the pleural effusion after the initiation of antituberculous treatment. High levels of ADA in the pleural fluid can be observed in patients with cryptococcal pleuritis, and longer incubation of pleural fluid should be performed in all patients who present with pleuritis associated with a high ADA level as the only significant finding.

\section{KARGER \\ Fax +41613061234 E-Mail karger@karger.ch} www.karger.com
(C) 2009 S. Karger AG, Basel 0025-7931/10/0792-0153\$26.00/0 www.karger.com/res
Takatoshi Kitazawa, MD, PhD

Department of Infectious Diseases, Graduate School of Medicine, University of Tokyo 7-3-1 Hongo, Bunkyo-ku

Tokyo 113-8655 (Japan)

Tel. +81 33815 5411, Fax +81 35800 8805, E-Mail tkitazaw-tky@umin.ac.jp 


\section{Introduction}

Cryptococcus neoformans is an important fungal pathogen causing serious infections in patients with acquired immune deficiency syndrome (AIDS) [1]. It is thought that the lungs are the initial site of almost all infections due to $C$. neoformans, which primarily affects the respiratory tract and central nervous system. However, pleural effusion alone is an unusual presentation of cryptococcal infection in AIDS patients [2-4]. We present a case of an AIDS patient with cryptococcal pleuritis who was initially diagnosed with tuberculous pleuritis because of the high level of adenosine deaminase (ADA) in the pleural fluid. An elevated ADA level in pleural fluid is usually associated with tuberculosis, although it can occasionally be seen in nontuberculous pleuritis, including cryptococcosis.

\section{Case Report}

A 51-year-old Japanese man suffered from productive cough for 4 months without presenting to an internist. He had a history of chronic hepatitis B infection and contacted his primary care doctor for a follow-up examination. A left pleural effusion was observed by chance when an abdominal ultrasound was performed. He was admitted to the University of Tokyo Hospital, Japan, for examination and treatment. He had been assigned to Myanmar for 4 years, and 11 months prior to admission, he had unprotected sexual contact.

On admission, his physical exam revealed a temperature of $36.9^{\circ} \mathrm{C}$, a pulse rate of $88 \mathrm{bpm}$ and a blood pressure of $146 / 90 \mathrm{~mm}$ $\mathrm{Hg}$. Breath sounds were attenuated on the left side of his chest, but neither rales nor murmurs were heard. There were no abnormalities on neurological examination such as neck stiffness or disori- entation. Laboratory findings on admission showed a white blood cell count of 5,300/ $\mathrm{ll}$ without a shift to the left and a C-reactive protein level of $7.33 \mathrm{mg} / \mathrm{dl}$. His admission chest X-ray film revealed a pleural effusion occupying two thirds of the left lung (fig. 1a) which was confirmed on chest CT. The chest CT was otherwise negative. Sputum culture was also negative. Acid fast bacilli stain and polymerase chain reaction test of his sputum for Mycobacterium tuberculosis were also negative. Thoracentesis and pleural biopsy were performed. No granulomatous lesions were histopathologically detected, and acid fast smear and culture of the biopsy sample were both negative. Analysis of the pleural effusion revealed an exudative fluid with a white blood cell count of 2,600/ $\mathrm{l}$ l and an ADA level of 85.9 IU/l. However, no microorganisms were detected in the culture of the pleural fluid after 1 week. Due to the high level of ADA in the effusion, a presumptive diagnosis of tuberculous pleuritis was made, and treatment with isoniazid, rifampicin, ethambutol and pyrazinamide, along with pleural drainage, was initiated.

After initiation of the antituberculous therapy, the pleural effusion gradually decreased, although the patient developed a fever. C. neoformans was detected in the culture of the pleural effusion by the 11th day of admission. A human immunodeficiency virus (HIV) antibody test was positive with $49 / \mu$ l of CD4+ T cells. The serum cryptococcal antigen was detected, although a quantification assay of the antigen was not available. Lumbar puncture was also performed. No cells were detected in the cerebrospinal fluid and the total protein was $23 \mathrm{mg} / \mathrm{dl}$, glucose was $52 \mathrm{mg} / \mathrm{dl}$, and acid fast bacilli stain and culture were negative. India ink staining showed no causative agents and the cryptococcal antigen test was negative.

Antituberculosis treatment was discontinued and amphotericin B $(0.7 \mathrm{mg} / \mathrm{kg} /$ day $)$ was administered for 2 weeks, followed by fluconazole therapy (400 mg/day) for 8 weeks. Four weeks after initiation of antifungal treatment, the pleural effusion resolved (fig. 1b). Six weeks after initiation of antifungal treatment, highly active antiretroviral therapy was started. On the 8th week of admission, the acid fast bacilli culture of the pleural effusion was negative. This patient has been well for 3 years without relapse of his pleuritis.
Fig. 1. Chest X-ray on admission (a) and 4 weeks after initiation of antifungal treatment (b).
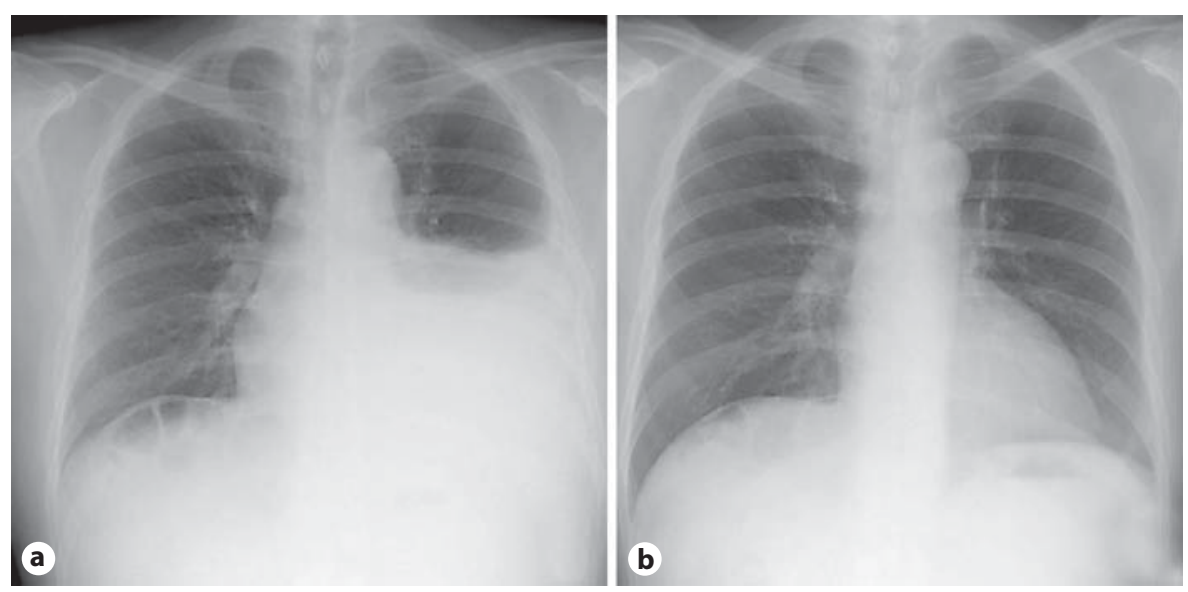


\section{Discussion}

Cryptococcal infection is the 4th most common opportunistic infection in patients with AIDS [1]. It is thought that the lungs are the initial site of almost all infections due to $C$. neoformans, and they are the second most clinically relevant site of infection after the central nervous system. However, pleural effusion is an unusual presentation of cryptococcal infection particularly in AIDS patients [2-4], and among 75 cases of HIV-infected patients with pleural effusion, only 4 cases were due to $C$. neoformans [5]. There have been few reports of AIDS patients presenting with cryptococcal pleuritis without other signs of infection $[3,6,7]$.

Therefore, our patient was initially diagnosed with tuberculous pleuritis based on the analysis of the pleural effusion which showed a high level of ADA, even though the culture of the pleural fluid was negative. In patients with tuberculous pleuritis, pleural fluid cultures are negative in more than $70 \%$ of patients, although cultures obtained from pleural biopsies are positive in $40-80 \%$ of cases $[8,9]$. Definitive diagnosis of tuberculosis pleural effusions depends on the demonstration of Mycobacterium tuberculosis in sputum, pleural fluid or pleural biopsy specimens. Most cases of pleural tuberculosis are diagnosed on the basis of cultures and histopathological findings $[8,10]$. Some cases are clinically diagnosed by supportive evidence alone, including demonstration of classical tuberculosis granulomas in the lung and elevated ADA and interferon- $\gamma$ levels in the pleural fluid. Previous studies have shown that levels of ADA in the pleural fluid $>40$ IU/l have a high sensitivity (81-100\%) and a high specificity (83-100\%) for tuberculosis pleuritis [8]. However, elevated levels of ADA in the pleural fluid can also be seen in empyema, lymphoma, other malignancies, parapneumonic effusions and pleural effusions associated with collagen vascular diseases $[11,12]$. Because $\mathrm{ADA}$ is the enzyme that catalyzes the conversion of adenosine to inosine, and ADA is found in most cells, particularly lymphocytes, it is conceivable that ADA would be elevated in lymphocyte-rich pleural effusions [13]. Cell fractionations of pleural fluid in previously reported cases of cryptococcal infection were lymphocyte rich $[3,14-17]$. In our case, the lymphocyte percentage of the pleural fluid could not be determined because cell fractionation analysis of pleural fluid was not available in our hospital. However, other studies showed that an elevated level of ADA was seldom found in nontuberculous lymphocytic pleural effusions, and an ADA level $<40$ IU/l virtually excluded tuberculosis in lymphocytic pleural effusions, although cases of cryptococcosis were not included in these studies $[18,19]$. In a study about tuberculous and nontuberculous pleuritis, evaluation of pleural ADA levels correlates with a CD4+ T lymphocyte population which is related to cellular immunity [20]. Other reported infectious diseases with high pleural ADA levels (other than tubercuosis) include legionellosis, brucellosis, coxiellosis and cryptococcosis [15, 21-23]. These are intracellular microbial agents, and their pathogenicity is related to cellular immunity [24-28]. There has been only 1 previous case of cryptococcosis where the ADA level in the pleural effusion was recorded as 27.8 IU/1 [15]. More investigation is required to further determine the association between cryptococcal pleuritis and the ADA level in pleural effusions.

Although it took 11 days to detect Cryptococcus in the culture of our patient's pleural effusion, and C. neoformans is usually cultured within 3-7 days, the culture time of pleural effusion can vary up to 2 weeks [3]. We assumed that more than 7 days would be required to culture pleural effusion in order to diagnose cryptococcosis.

In conclusion, high levels of ADA in the pleural fluid can be observed in patients with cryptococcal pleuritis, and a longer incubation of pleural fluid should be performed in all patients who present with pleuritis as the only significant finding.
References

\footnotetext{
1 Kovacs JA, Kovacs AA, Polis M, Wright WC, Gill VJ, Tuazon CU, Gelmann EP, Lane HC Longfield R, Overturf G, et al: Cryptococcosis in the acquired immunodeficiency syndrome. Ann Intern Med 1985;103:533-538.

2 Grum EE, Schwab R, Margolis ML: Cryptococcal pleural effusion preceding cryptococcal meningitis in AIDS. Am J Med Sci 1991; 301:329-330.
}

\footnotetext{
-3 Katz AS, Niesenbaum L, Mass B: Pleural effusion as the initial manifestation of disseminated cryptococcosis in acquired immune deficiency syndrome. Diagnosis by pleural biopsy. Chest 1989;96:440-441.

4 Young EJ, Hirsh DD, Fainstein V, Williams TW: Pleural effusions due to Cryptococcus neoformans: a review of the literature and report of two cases with cryptococcal antigen determinations. Am Rev Respir Dis 1980; 121:743-747.
} 
5 Cadranel JL, Chouaid C, Denis M, Lebeau B, Akoun GM, Mayaud CM: Causes of pleural effusion in $75 \mathrm{HIV}$-infected patients. Chest 1993; 104:655.

6 Garcia Garcia JC, Baloira Villar A, Anibarro Garcia L, Rodriguez Garcia JC, de Lis Munoz JM, Otero Portela JL: Pleural effusion as the only manifestation of cryptococcosis in an AIDS patient. Arch Bronconeumol 1994;30: 166-169.

7 Mauri M, Fernandez Sola A, Capdevila JA, Ruiz I, Ribera E, Ocana I, Ferrer A, Pahissa A: Pleural cryptococcosis in patients with human immunodeficiency virus infection. Med Clin (Barc) 1996;106:380-382.

8 Gopi A, Madhavan SM, Sharma SK, Sahn SA: Diagnosis and treatment of tuberculous pleural effusion in 2006. Chest 2007;131: $880-889$.

$\checkmark 9$ Froudarakis ME: Diagnostic work-up of pleural effusions. Respiration 2008;75:4-13.

-10 Liam CK, Lim KH, Wong CM: Tuberculous pleurisy as a manifestation of primary and reactivation disease in a region with a high prevalence of tuberculosis. Int J Tuberc Lung Dis 1999;3:816-822.

-11 Ocana I, Ribera E, Martinez-Vazquez JM, Ruiz I, Bejarano E, Pigrau C, Pahissa A: Adenosine deaminase activity in rheumatoid pleural effusion. Ann Rheum Dis 1988; 47:394-397.
2 Burgess LJ, Maritz FJ, Le Roux I, Taljaard JJ: Combined use of pleural adenosine deaminase with lymphocyte/neutrophil ratio. Increased specificity for the diagnosis of tuberculous pleuritis. Chest 1996;109:414-419.

13 Ungerer JP, Oosthuizen HM, Bissbort SH, Vermaak WJ: Serum adenosine deaminase: isoenzymes and diagnostic application. Clin Chem 1992;38:1322-1326.

14 Newman TG, Soni A, Acaron S, Huang CT: Pleural cryptococcosis in the acquired immune deficiency syndrome. Chest 1987;91: 459-461.

15 Fukuchi M, Mizushima Y, Hori T, Kobayashi M: Cryptococcal pleural effusion in a patient with chronic renal failure receiving longterm corticosteroid therapy for rheumatoid arthritis. Intern Med 1998;37:534-537.

$>16$ Epstein R, Cole R, Hunt KK Jr: Pleural effusion secondary to pulmonary cryptococcosis. Chest 1972;61:296-298.

17 Houk VN, Moser KM: Pulmonary cryptococcosis. Must all receive amphotericin B? Ann Intern Med 1965;63:583-596.

-18 Jimenez Castro D, Diaz Nuevo G, PerezRodriguez E, Light RW: Diagnostic value of adenosine deaminase in nontuberculous lymphocytic pleural effusions. Eur Respir J 2003;21:220-224.

19 Lee YC, Rogers JT, Rodriguez RM, Miller KD, Light RW: Adenosine deaminase levels in nontuberculous lymphocytic pleural effusions. Chest 2001;120:356-361.

-20 Baganha MF, Pego A, Lima MA, Gaspar EV, Cordeiro AR: Serum and pleural adenosine deaminase. Correlation with lymphocytic populations. Chest 1990;97:605-610.
21 Dikensoy O, Fakili F, Elbek O, Uysal N: High adenosine deaminase activity in the pleural effusion of a patient with Legionnaires' disease. Respirology 2008;13:473-474.

-22 Esteban C, Oribe M, Fernandez A, Ramos J, Capelastegui A: Increased adenosine deaminase activity in $\mathrm{Q}$ fever pneumonia with pleural effusion. Chest 1994;105:648.

-23 Dikensoy O, Namiduru M, Hocaoglu S, Ikidag B, Filiz A: Increased pleural fluid adenosine deaminase in brucellosis is difficult to differentiate from tuberculosis. Respiration 2002;69:556-559.

-24 Rodrigues ML, Alviano CS, Travassos LR: Pathogenicity of Cryptococcus neoformans: virulence factors and immunological mechanisms. Microbes Infect 1999;1:293-301.

>25 Reimer LG: Q fever. Clin Microbiol Rev 1993;6:193-198.

26 Golding B, Scott DE, Scharf O, Huang LY, Zaitseva M, Lapham C, Eller N, Golding H: Immunity and protection against Brucella abortus. Microbes Infect 2001;3:43-48.

27 Horwitz MA: Cell-mediated immunity in Legionnaires' disease. J Clin Invest 1983;71: 1686-1697.

28 Splitter G, Oliveira S, Carey M, Miller C, Ko J, Covert J: T lymphocyte mediated protection against facultative intracellular bacteria. Vet Immunol Immunopathol 1996;54: 309-319. 